\title{
Factors Influencing Recovery from Fatigue after a 16-hour Night Shift among Female Japanese Nurses
}

\author{
Narumi Ooshige $^{1^{*}}$, Tae Yamaguchi ${ }^{2}$, Mitsuyo Nakashima ${ }^{3}$, Kazuo Minematsu $^{4,5}$, Takuro Tobina ${ }^{6}$ and Noriaki Tsunawake \\ ${ }^{1}$ Japanese Red Cross Kyushu International College of Nursing, 1-1 Asty Munakata, Fukuoka, 811-4157, Japan \\ ${ }^{2}$ Department of Nursing, Faculty of Nursing and Nutrition, University of Nagasaki,1-1-1Manabino, Nagayo-cyo, Nishisonoki-gun, Nagasaki, 851- \\ 2195, Japan \\ ${ }^{3}$ Faculty of Medicine, School of Nursing, Fukuoka University, 8-19-1 Nanakuma, Jonan-ku, Fukuoka, 814-0180, Japan \\ ${ }^{4}$ Graduate School of Education, Nagasaki University, 1-14 Bunkyomachi, Nagasaki, 852-8521, Japan \\ ${ }^{5}$ Department of Public Health, School of Medicine, Juntendo University, 2 Chome-1-1 Hongo, Bunkyo-ku, Tokyo 113-0033, Japan \\ ${ }^{6}$ Faculty of Nursing and Nutrition, University of Nagasaki,1-1-1Manabino, Nagayo-cyo, Nishisonoki-gun, Nagasaki, 851-2195, Japan
}

\begin{abstract}
Background: The present study aimed to evaluate self-reported fatigue recovery among Japanese nurses who perform shift work and to evaluate how their fatigue was affected by a day off after a night shift.

Methods: We recruited 66 healthy female Japanese nurses at three regional private hospitals. The nurses were divided into a recovery group and a non-recovery group, based on their self-reported experience of fatigue during the interval from the time before their night shift to after a following day off. Unpaired t-test and logistic regression analyses were used to find associations of self-reported fatigue with demographic and lifestyle factors.

Results: The recovery group included 45 nurses, and the non-recovering group included 21 nurses. The recovery group exhibited a high degree of fatigue at five assessment points, while the non-recovery group only exhibited a high degree of fatigue at the post-day off assessment. The adjusted odds ratios (95\% confidence intervals) for self-reported fatigue during the night shift were $1.27(1.00-1.61)$ for the age of the nurse's youngest child and $1.04(1.00-1.08)$ for the nurse's time spent napping $(\mathrm{P}<0.05)$.
\end{abstract}

Conclusion: Sleeping before a night shift may facilitate recovery after the shift, although fatigue was also influenced by the age of the nurse's youngest child and the nurse's napping time.

\section{Background}

Nurses work in a dynamic environment [1], and the risk of accidents increases among nurses who completeconsecutive night shifts [2]. Shift work also contributes to impaired sleep in other occupations, including both high- and low-stress occupations [3]. Among shift workers, social support from supervisors is important [4], and nurses' performance is affected by their working conditions and level of fatigue [5], which can be directly related to poor working conditions. For shift workers, life attitudes and practices that do not leave fatigue are important to improve performance.

In Japan, nurses' work uses a two- or three-shift rotation system; the two-shift rotation system has been adopted by $57.4 \%$ of Japanese hospitals, although it is associated with increased health risks compared to the three-shift system [6]. To help address this, nurses who perform shift work should receive a day off after a night shift to recover from work-related fatigue [7]. Active and fulfilling non-work activities can promote a healthy lifestyle, reduce stress, and reduce feelings of chronic fatigue[8]. Therefore, it is important to spend days off wisely to recover from night shifts.

Females account for $93 \%$ of all Japanese nurses [9]. As a result, it increases that female nurses experience more shift work than the male nurses. Moreover, the female nurses are playing the roles not only of nursing business but the housework in many cases. Furthermore, age, napping time during a night shift, physical activity level, and lifestyle are strongly associated with fatigue during a night shift; however, a comprehensive analysis of these factors, including psychological research, has not been conducted [10]. As such, the present study aimed to evaluate the degree of self-reported fatigue among female.
Japanese nurses who performed shift work and to evaluate how their fatigue was affected by a day off after a night shift. This study is intended to contribute to reducing fatigue in female nurses after 16hour night shifts with a two-shift rotation system

\section{Materials and Methods}

\section{Participants}

We recruited 66 healthy female Japanese nurses from three regional private hospitals in the northern Kyushu area of Japan. The nurses were divided into a recovery group and a non-recovery group based on their self-reported experience of fatigue during the interval from the time before their night shift to after a day off.

\section{Data collection}

Between June 2014 and April 2016, we collected data regarding the nurses' demographics (age, family members, age of their youngest child, and commuting time), years of nursing experience, years of

"Corresponding Author: Dr. Narumi Ooshige, Japanese Red Cross Kyushu International College of Nursing, 1-1 Asty Munakata, Fukuoka, 811-4157, Japan, Tel: 098-895-1269; E-mail: n-ooshige@jrckicn.ac.jp

Citation: Ooshige N, Yamaguchi T, Nakashima M, Minematsu K, Tobina T, et al. (2017) Factors Influencing Recovery from Fatigue after a 16-hour Night Shift among Female Japanese Nurses. Int J Nurs Clin Pract 4: 255. doi: https://doi. org/10.15344/2394-4978/2017/255

Copyright: (c) 2017 Ooshige et al. This is an open-access article distributed under the terms of the Creative Commons Attribution License, which permits unrestricted use, distribution, and reproduction in any medium, provided the original author and source are credited. 
Citation: Ooshige N, Yamaguchi T, Nakashima M, Minematsu K, Tobina T, et al. (2017) Factors Influencing Recovery from Fatigue after a 16-hour Night Shift among Female Japanese Nurses. Int J Nurs Clin Pract 4: 255. doi: https://doi.org/10.15344/2394-4978/2017/255

Page 2 of 5

experience of working night shifts, lifestyle, and napping time. Selfreported fatigue was measured based on subjective fatigue symptoms, evaluated at six points,before and after day shift, before and after a night shift, and before and after a day off. This was done using the Jikaku-sho Shirabe questionnaire, as recommended by the Working Group for Occupational Fatigue [11]. Responses for each component range from 0 (totally disagree) to 5 (agree strongly), with higher scores indicating worse symptoms. Each subjective symptom (drowsiness, instability, uneasiness, dullness, and eyestrain) is evaluated using five questions, and the maximum possible score for each subjective symptom is 25 . Recovery was defined as the individual's score returning to its pre-stressor level (i.e., the effects of the stressor were subjectively reduced), and the critical measurement points for fatigue were defined as the time before the night shift and after a day off. Fatigue was also assessed using the Cumulative Fatigue Symptoms Index, which includes 81 items in eight categories: decreased vitality, general fatigue, physical disorders, irritability, decreased willingness to work, anxiety, depressive feelings, and chronic tiredness[12].Physical activity levels (steps and energy expenditure (EE) from before night shift to after day off) were examined using a three-dimensional speed sensor. Physical activity levels were measured by Actimaker(EW4800, Panasonic, Japan).

\section{Ethical considerations}

The study protocol was approved by the ethics committee of the Japanese Red Cross Kyushu International College of Nursing (Fukuoka, Japan). The purpose and procedures of this study were explained to the directors of each hospital, and all nurses provided their informed consent before participating in the study.

\section{Statistical analysis}

Differences between the recovery and non-recovery groups were evaluated using the unpairedt-test for continuous variables (age,age of the youngest child, years of nursing experience, years of experience of working night shifts, commuting time, napping time, sleeping hours, and the Cumulative Fatigue Symptoms Index score). Values were generally reported as mean \pm standard deviation. Logistic regression analyses were used to investigate factors that were associated with fatigue after working night shifts.Differences were considered statistically significant at a P-value of $<0.05$, and all analyses were performed using SPSS software (version 23.0; SPSS Japan, Tokyo, Japan).

\section{Results}

\section{Participant characteristics}

the non-recovery group, 13nurses(61.9\%) had children, eight nurses(38.1\%) did not have children, 15 nurses (71.4\%) were living with their family, and six nurses $(28.6 \%)$ were not living with their family. There was no significant difference in the nurses' average ages when we compared the recovery and non-recovery groups (38.2 years vs. 38.1 years, respectively). The recovery group had a relatively young age for the youngest children compared to the non-recovery group (3.4 years vs. 7.9 years, respectively), although this difference was not statistically significant. There were also no significant differences between the recovery and non-recovery groups in theiryears of experience of working night shifts and commuting times. The recovery group exhibited a high degree of fatigue at all six assessment points between their night shift and day off, whereas the non-recovery group

\section{exhibited a highdegree of fatigue only at the assessment on a day off.}

The recovery group had an average napping time of $55.6 \mathrm{~min}$ compared to $85.2 \mathrm{~min}$ in the non-recovery group, although this difference was not statistically significant. The two groups also did not exhibit significant differences in the number of sleeping hours for day shifts and night shifts. However, before a night shift, the nonrecovery group exhibited a shorter average sleeping time compared to the recovery group ( $425.0 \mathrm{~min}$ vs. $506.3 \mathrm{~min}$, respectively; $\mathrm{P}<$ $0.05)$. The recovery group also exhibited lower Cumulative Fatigue Symptoms Index scores for anxiety and chronic fatigue compared to the non-recovery group $(\mathrm{P}<0.05)$. The only significant difference in day-off activities between the recovery and non-recovery groups was observed in the number of sleeping hours $(\mathrm{P}<0.05)$ (Table1).

\section{Factors associated with recovery from fatigue among female Japanese night shift nurses}

Logistic regression analyses were used to calculate the odds ratios for factors that might be associated with recovery from fatigue among female Japanese nurses (age, years of experience working night shifts, number of children, age of youngest child, commuting time, sleeping hours after a day shift, sleeping hours before a night shift, napping time, sleeping hours after a night shift, sleeping hours during the day off, anxiety score, and general fatigue score). The adjusted odds ratios for fatigue during the night shift among the female Japanese nurses were 1.27 (95\% confidence interval: 1.00-1.61) for the age of the youngest child and 1.04 (95\% confidence interval: 1.00-1.08) for the nurses' napping time $(\mathrm{P}<0.05)$. The age of the youngest child and the nurses'napping time were associated with recovery from fatigue after working night shifts (Table 2).

\section{Discussion}

In shift work systems, sufficient sleep hours are needed for nurses who work night shift to ensure good quality of sleep and consequently better services for patients [13]. The present study did not detect significant differences between the recovery and non-recovery groups in terms of age, years of experience of working night shifts, or commuting time. However, the recovery group exhibited high fatigue during the evaluation period, and as the recovery group exhibited changes in their level of fatigue, recovery from fatigue was easily observed. It appears that recovery from fatigue (accumulated between day shifts)can be achieved through appropriate night-time sleeping [14] but is not achieved if sleeping hours are insufficient. Interestingly, the recovery group had more stable times to ensure appropriate sleeping time compared to the non-recovery group during days off and before/after night shifts. For example, the recovery group exhibited longer sleeping time before a night shift. A previous study evaluated participants who completed four consecutive night shifts and then received a day off before returning to day shifts. The study revealed that persons who achieved early fatigue recovery exhibited high-quality night-time sleeping and earlier times going to sleep [15]. Thus, our findings may be related to the nurses in the recovery group being aware of their fatigue, and adjusting their sleep timing and duration accordingly.

The recovery group exhibited higher scores for anxiety and chronic fatigue compared to the non-recovery group. Anxiety can be related to both mental fatigue and general work-related fatigue [16], and somatic symptoms are useful for evaluating female nurses who perform 
Citation: Ooshige N, Yamaguchi T, Nakashima M, Minematsu K, Tobina T, et al. (2017) Factors Influencing Recovery from Fatigue after a 16-hour Night Shift among Female Japanese Nurses. Int J Nurs Clin Pract 4: 255. doi: https://doi.org/10.15344/2394-4978/2017/255

Page 3 of 5

\begin{tabular}{|c|c|c|c|c|c|c|}
\hline & & \multicolumn{2}{|c|}{ Recovery group $(n=45)$} & \multicolumn{2}{|c|}{ Non-recovery group $(\mathrm{n}=21)$} & \multirow[b]{2}{*}{$p$-value } \\
\hline & & Mean & SD & Mean & SD & \\
\hline Age & & 38.2 & 9.5 & 38.1 & 10.1 & 0.981 \\
\hline The age of youngest child(age) & & 3.4 & 4.4 & 7.9 & 8.5 & 0.058 \\
\hline Years of clinical experience on the night shift & & 13.2 & 9.2 & 11.5 & 10.9 & 0.515 \\
\hline Number of children & & 0.8 & 1.2 & 1.1 & 0.8 & 0.464 \\
\hline Commuting time (min) & & 22.5 & 13.4 & 21.9 & 10.9 & 0.862 \\
\hline \multirow[t]{6}{*}{ The degree of self-conscious fatigue } & Before on a day shift & 39.1 & 27.0 & 35.0 & 24.5 & 0.558 \\
\hline & After on a day shift & 43.9 & 30.2 & 37.9 & 27.5 & 0.437 \\
\hline & Before night shift & 40.5 & 25.9 & 30.9 & 23.3 & 0.152 \\
\hline & After night shift & 52.6 & 29.9 & 50.3 & 30.7 & 0.772 \\
\hline & Before on a day shift & 33.0 & 16.9 & 30.4 & 17.6 & 0.562 \\
\hline & After on a day shift & 38.0 & 11.3 & 50.3 & 20.7 & 0.003 \\
\hline Nap time (min) & & 65.6 & 46.1 & 85.2 & 53.1 & 0.133 \\
\hline \multirow[t]{3}{*}{ Sleeping hour } & After on a day shift (min) & 581.0 & 70.2 & 352.6 & 64.0 & 0.137 \\
\hline & Before night shift (min) & 5.06 .3 & 124.5 & 425.0 & 83.6 & 0.009 \\
\hline & After night shift (min) & 468.8 & 127.6 & 433.8 & 103.6 & 0.242 \\
\hline \multirow[t]{8}{*}{ Rate of CSFI } & Decreased vitality & 37.9 & 29.9 & 41.6 & 31.4 & 0.644 \\
\hline & General fatigue & 37.1 & 26.1 & 37.2 & 24.9 & 0.981 \\
\hline & Physical disorders & 28.3 & 22.5 & 26.5 & 22.7 & 0.774 \\
\hline & irritability & 24.1 & 24.7 & 25.9 & 28.1 & 0.801 \\
\hline & Unwillingness to work & 25.1 & 24.1 & 25.3 & 30.2 & 0.984 \\
\hline & anxiety & 35.0 & 31.5 & 19.5 & 25.6 & 0.039 \\
\hline & Depressive feeling & 35.1 & 27.4 & 24.9 & 26.7 & 0.061 \\
\hline & Chronic fatigue & 58.1 & 29.0 & 38.1 & 36.8 & 0.020 \\
\hline \multirow[t]{8}{*}{ The life activities of a day off } & Sleeping time (min) & 501.0 & 117.4 & 432.7 & 87.7 & 0.021 \\
\hline & Eating time (min) & 82.5 & 35.1 & 86.6 & 33.9 & 0.656 \\
\hline & Working time (min) & 18.3 & 54.1 & 44.0 & 102.4 & 0.291 \\
\hline & Housekeeping (min) & 323.1 & 201.8 & 428.8 & 209.5 & 0.055 \\
\hline & $\begin{array}{l}\text { Conversation and } \\
\text { relations }(\mathrm{min})\end{array}$ & 76.7 & 130.7 & 66.2 & 126.6 & 0.759 \\
\hline & Leisure (min) & 141.2 & 195.1 & 106.7 & 133.0 & 0.466 \\
\hline & Mass media (min) & 238.9 & 174.9 & 210.7 & 135.1 & 0.517 \\
\hline & Rest (min) & 58.3 & 85.2 & 64.4 & 59.3 & 0.769 \\
\hline
\end{tabular}

Table 1: Comparison of the characteristics affecting fatigue in the recovery and non-recovery groups.

Comparison of the characteristics affecting fatigue in the recovery and non-recovery groups, calculated by unpaired $t$-test.

\begin{tabular}{|c|c|c|c|c|c|}
\hline & \multicolumn{5}{|c|}{ OR of $95 \%$ confidence interval } \\
\hline & Or (odds ratio) & Minimum & Maximum & VIF & $P$-value \\
\hline age & 0.89 & 0.69 & 1.14 & 6.03 & 0.349 \\
\hline Years of clinical experience on the night shift & 1.02 & 0.83 & 1.25 & 3.91 & 0.871 \\
\hline Number of children & 0.85 & 0.20 & 3.67 & 2.56 & 0.824 \\
\hline The age of the youngest child (age) & 1.27 & 1.00 & 1.61 & 2.11 & 0.049 \\
\hline Commuting time(min) & 1.00 & 0.87 & 1.15 & 2.16 & 0.998 \\
\hline Sleeping hours after on a day shift & 0.99 & 0.97 & 1.01 & 1.72 & 0.245 \\
\hline Sleeping hours before night shift & 1.00 & 0.98 & 1.01 & 2.53 & 0.752 \\
\hline Nap time (min) & 1.04 & 1.00 & 1.08 & 1.21 & 0.039 \\
\hline Sleeping hour after night shift & 1.00 & 0.99 & 1.01 & 1.94 & 0.882 \\
\hline Sleeping hour of an day off & 0.99 & 0.98 & 0.01 & 1.77 & 0.291 \\
\hline Rate of anxiety & 0.94 & 0.88 & 1.01 & 2.57 & 0.110 \\
\hline Rate of general fatigue & 1.02 & 0.97 & 1.08 & 2.02 & 0.370 \\
\hline constant & 11176.69 & & & & 0.165 \\
\hline
\end{tabular}

Table 2: Factors associated with recovery from fatigue among female Japanese night nurses.

Dummy variable: The recovery group; 1 the non-recovery group; 2

Factor associated with recovery from fatigue among female Japanese night shift nurses, identified by logistic regression analyses. 
Citation: Ooshige N, Yamaguchi T, Nakashima M, Minematsu K, Tobina T, et al. (2017) Factors Influencing Recovery from Fatigue after a 16-hour Night Shift among Female Japanese Nurses. Int J Nurs Clin Pract 4: 255. doi: https://doi.org/10.15344/2394-4978/2017/255

Page 4 of 5

various tasks and their feelings regarding fatigue; however, their physical condition is a reflection of their emotional condition, and the recovery group usually exhibited greater feelings of fatigue compared to the non-recovery group. Therefore, the recovery group likely experienced significant insecurity and feelings of chronic fatigue. In the logistic regression analyses, the age of the youngest child and the nurses' napping time were associated with recovery from fatigue after a night shift. A younger age for the youngest child (in the recovery group, a mean of 3.4 years) resulted in longer sleeping hours on the day off; these longer sleeping hours are presumed to influence recovery from fatigue. Since the child is young, the female nurses of the recovery group may only be sleeping early together Thus, a continuous break of at least $60 \mathrm{~min}$ is needed to facilitate an appropriate nap after a night shift [17].

In the present study, the non-recovery group exhibited an average napping time of 85.2 minutes, which was longer than the napping time for the recovery group. However, previous research indicates that nurses who work 16-h night shifts need an average napping time of $2.33 \mathrm{~h}$, which is greater than the $2 \mathrm{~h}$ that was allocated to these individuals [18]. Furthermore, as alertness remains suboptimal in the $15 \mathrm{~min}$ after awakening, nurses should not perform important tasks during this period as they have a high risk of accidents, even if the individual subjectively believes that they are not sleepy [19]. Therefore, long naps are used to minimize the state of suboptimal alertness, although the decline in post-nap sleepiness during a night shift is not correlated with the feeling of having done a good job [20].

An older age for the youngest child (in the non-recovery group, a mean of 7.9 years) was associated with poorer fatigue recovery after a night shift, and a previous study found that having preschool children was associated with greater fatigue compared to having school-age children [21]. In this context, mothers of preschool children are expected to perform more housework and have less time for sleeping and social activities, which presumably influences fatigue. However, nurses without children are more likely to experience job turnover [22], and nurses with children may receive more support from their family. Nevertheless, workers with preschool-aged children need support in adjusting to work and adapting their sleep patterns to their individual conditions [23]. Moreover, these workers may not get sufficient sleep, even on weekends, because of interruptions to sleep caused by their children [24]. Although the length and timing of naps varied widely among the previous studies, 30-40-min nap opportunities have been recommended between 02:00 and 03:00 [25] Thus, although the effects of child-rearing are not clearly established, the child's age seems to affect the process of recovery from fatigue.

This study had several limitations. First, its limited sample size and small control group are associated with known risks of bias. Second, the continuous wearing of an activity monitor may not have been completed on the days off. Therefore, the activity monitor measurements might not have been consistent. An important point, however, that the study establishes is that to recover from fatigue after shift work, it is necessary to sleep before night shifts and that nurses with small children especially need to take sufficient naps.

\section{Conclusions}

In conclusion, the present study revealed that recovery from fatigue after a night shift appears to be affected by the nurses' life activities and circumstances, such as sleeping before a night shift, nap duration, and the age of the youngest child.

\section{Funding}

This research was supported by a Grant-In-Aid for Scientific Research from the Japan Society for the Promotion of Science (No. 26463295).

\section{Competing Interests}

The authors declares that they have no competing interests.

\section{Author Contributions}

Narumi Ooshige, Tae Yamaguchi, Mitsuyo Nakashima, Takuro Tobina, Kazuo Minematsu, and Noriaki Tsunawaki were responsible for the study conception and design and for critical revisions to the paper for important intellectual content. Narumi Ooshigeand Tae Yamaguchi performed the data collection. Narumi Ooshige, Mitsuyo Nakashima, Takuro Tobina, Kazuo Minematsu, and Noriaki Tsunawakei were responsible for the drafting of the manuscript and for statistical analysis of the data. Narumi Ooshige obtained funding and supervised the study.

\section{References}

1. Tawil FA (2015) Low back pain and patients lifting behaviors among nurses working in Prince Abdel Rahman AlSadairy Hospital, Saudi Arabia. Int J Adv Res 3: 1211-1223.

2. Rogers AE (2008) The Effects of Fatigue and Sleepiness on Nurse Performance and Patient Safety. In: Hughes RG (Ed) Patient Safety and Quality: An Evidence-Based Handbook for Nurses, Agency for Healthcare Research and Quality, USA, pp. 1-37.

3. Karhula K, Harma M, Sallinen M, Hublin C, Virkkala J, et al. (2013) Job strain, sleep, and alertness in shiftworking health care professionals: a field study. Ind Health 51: 406-416.

4. Pisarski A, Bohle P (2001) Effects of supervisor support and coping on shiftwork tolerance. J Hum Ergol (Tokyo) 30: 363-368.

5. Ahmed MF, Sleem WF, Kassem AH (2015) Effect of working condition and fatigue on performance of staff nurses at Mansoura University hospital. J Nurs Health Sci 4: 83-91

6. Japanese Nursing Association (2014) A report about the spread of the night shift and rotation shiftwork guidelines for nurses.

7. Kubo T, Takahashi M, Sallinen M, Kubo Y, Suzumura H (2013) How are leisure activity and shiftwork schedule associated with recovery from fatigue in shiftwork nurses? Sangyo Eiseigaku Zasshi 55: 90-102.

8. Shao MF, Chou YC, Yeh MY, Tzeng WC (2010) Sleep quality and quality of life in female shift-working nurses. J Adv Nurs 66: 1565-1572.

9. Ministry of Health, Labour and Welfare (2014) General condition of the example of the Heisei 26 public-health-administration report (employment medical staff).

10. Minematsu $\mathrm{K}$, Yamaguchi $\mathrm{T}$, Nakashima $\mathrm{M}$, Tsunawake $\mathrm{N}$, Ooshige $\mathrm{N}$ (2017) Health management for medical-surgical female nurses having nightshift working. Int J Nurs Clin Pract 4: 216-221.

11. Tachi N (2002) The proposal and revised work progress of a new edition of “Jikaku-sho Shirabe. J Sci Labor 57: 299-304.

12. Kosugo R, Fujii H (2009) Harmony of labor and health: CFSI manual. Institute for the Science of Labour 106-139

13. Ohida T, Kamal A, Sone T, Ishi T, Uchiyama M, et al. (2001) Night-shiftwork related problems in young female nurses. J Occup Health43: 150-156.

14. Onoue H (2004) Sleep and Fatigue. In: Hirou no Kagaku, Koudansha, Japan, pp. 14-15

15. Kubo T, Takahashi M, Tachi N, Takeyama H, Ebara T, et al. (2009) Characterizing recovery of sleep after four successive night shifts. Ind Health 47: 527-532. 
Citation: Ooshige N, Yamaguchi T, Nakashima M, Minematsu K, Tobina T, et al. (2017) Factors Influencing Recovery from Fatigue after a 16-hour Night Shift among Female Japanese Nurses. Int J Nurs Clin Pract 4: 255. doi: https://doi.org/10.15344/2394-4978/2017/255

16. Kikuchi Y, Ishii N (2015) The relationship between self-awareness of fatigue symptoms and working conditions in female nurses. Sangyo Eiseigaku Zasshi 57: 230-240.

17. Alger SE, Lau H, Fishbein W (2012) Slow wave sleep during a daytime nap is necessary for protection from subsequent interference and long-term retention. Neurobiol Learn Mem 98: 188-196.

18. Oriyama S, Miyakoshi $Y$, Kobayashi T (2014) Ways of taking rest and breaks related to night shifts in two-shift nurses and factors supporting work: a comparison of $12-$ and 16 -hour night shifts. J Jpn Soc Healthc Admin 51: 21-31

19. Milter MA, Carskadon MA, Czeisler CA, Dement WC, Dinges DF, et al (1988) Catastrophes, sleep, and public policy: consensus report. Sleep11: 100-109.

20. Barthe B, Tirilly G, Gentil C, Toupin C (2016) Job demands and resting and napping opportunities for nurses during night shifts: impact on sleepiness and self-evaluated quality of healthcare. Ind Health 54: 157-162.

21. Tounai M, Tounai S (2004) Time study of nurses' shiftwork and the influence of domestic labor on fatigue after ordinary labor. Jpn J Public Health 51: 874-883.

22. Arakawa C (2011) Factors affecting the resignation of female nurses: longitudinal study of nurses working at hospitals with more than 200 beds in the general ward in the Kanto region. J Jpn Soc Nurs Res 34: 85-92.

23. Sasaki T, Matsumoto S (2005) Actual conditions of work, fatigue, and sleep in non-employed, home-based female information technology workers with preschool children. Ind Health 43: 142-150.

24. Watanabe E, Aizawa R, Shiga K, Kanbayashi T, Takahashi K (2002) The quality of sleep in women who are working in daytime and taking care of infants during nights. Jpn Red Cross Jr Coll Akita 6: 43-46.

25. Ruggiero JS, Redeker NS (2014) Effects of napping on sleepiness and sleep-related performance deficits in night-shiftworkers: a systematic review. Biol Res Nurs 16: 134-142. 